American Journal of Applied Sciences 2 (10): 1380-1388, 2005

ISSN 1546-9239

(c) 2005 Science Publications

\title{
Balancing Treatment and Prevention: The Case of HIV/AIDS
}

\author{
Joseph Y.T. Mugisha \\ Department of Mathematics, Makerere University, P. O. Box 7062, Kampala, Uganda
}

\begin{abstract}
A mathematical model is formulated to study the effect of treatment of HIV/AIDS patients on the spread of the epidemic in a two-age group's population. The model assumes the sexual transmission mode. A proportion $\delta$, of the adult HIV/AIDS infects is assumed to be receiving treatment. The analysis of the model shows that treatment that is not accompanied by a positive change in social behavior will increase the number of both child and adult infections in the population. And if treatment is accompanied by a change in social behavior the epidemic could be eventually contained. The basic reproductive numbers in the case of treatment with no behavioral change and in the case treatment with behavioral change are used to make conclusions about the need to balance treatment with prevention.
\end{abstract}

Key words: Treatment, opportunistic infections, behavioral change, HIV/AIDS

\section{INTRODUCTION}

HIV/AIDS at present has no cure and no vaccine as yet. As the number of reported cases of AIDS continues to swell, the magnitude and severity of the problem become increasingly evident and the need to develop effective strategies to prevent and control HIV infection are becoming even more urgent. The virus is associated with a severe breakdown of the immune system of the affected person, rendering the body to be immunedeficient and these results in catastrophic susceptibility of the person infected with HIV/AIDS to opportunistic infections. These infections are not due to HIV/AIDS to say. They are common infections that do take a chance of a rather weakened and incompetent immune system and occasionally invade the patient with little or no resistance.

In many cases, an HIV-infected individual may be invaded by a host of common diseases ranging from very strong bacterial and viral infections to mild sicknesses. Some of the most common AIDS-related opportunistic infections that lead to AIDS-related morbidity and mortality is the pulmonary infections, such as Tuberculosis (TB), Pneumoccystis carinnii (pneumonia), the central nervous disorders like encephalitis, meningitis and the gastrointestinal tract infections ${ }^{[1-6]}$.

Since the infected person may be invaded by many infections simultaneously, it is noted that a single drug may not be sufficient to clear such an attack. (For example, an individual who has been diagnosed with TB may be found with herpes zoster and with persistent vomiting). As a result, a couple of drug recombinants that are particularly made to treat the HIV/AIDS patients of combinations of attacks have been developed $^{[7-10]}$.

This therefore brings us to the role of treatment of HIV/AIDS patients on the spread of the epidemic.
Many authors ${ }^{[11-21]}$ have studied the effect of treatment of HIV/AIDS patients, the role of the increased incubation period and behavioral change on the spread of the epidemic. The major result of this is that there is evidence that as awareness of the risks associated with HIV infection increases, there is a change in social behavior and also that therapy with behavioral change alters the course of the HIV epidemic. However there is little attempt in modeling treatment of HIV patients with age-structured population.

In this study, a population is divided into two age groups. The group I comprises of sexually immature children age a year and Group II comprises of sexually mature and active adults aged $a$ years and beyond. It is Group II that is responsible for the spread of the epidemic through sexual activity and for the spread in the children's group through infected mothers (vertical transmission). Treatment of opportunistic infections is done in the adult group and the model is qualitatively and numerically analyzed to determine the effect of the spread of the epidemic on the children and on the adult populations vis-à-vis change in social behavior.

\section{THE MODEL}

Notation and definitions: We use the following notations and/or definitions:

Susceptible: an individual who has not yet contracted the HIV but may contract it if exposed to any mode of its transmission.

Infect: an individual who has contracted the HIV and is capable of transmitting it; (also herein referred to as an Infected).

AIDS cases: infected individuals who have shown fullblown symptoms. 
Incubation period: the period of time it takes an individual who has acquired HIV to develop or show full-blown AIDS symptoms.

Let the Susceptibles, Infectives (HIV infected) and AIDS cases in Group I and II be $\mathrm{S}_{\mathrm{i}}(\mathrm{t}), \mathrm{I}_{\mathrm{i}}(\mathrm{t})$ and $\mathrm{A}_{\mathrm{i}}(\mathrm{t})$, i $=1,2$; respectively. Define the following parameters:

$\lambda$ : $\quad$ The per capita birth rate of Group II

$\mu_{\mathrm{i}}$ : The per capita AIDS-free mortality rate of Group i

$\mathrm{v}_{\mathrm{i}}$ : The rate at which HIV infected (or infects) progress to AIDS in Group i

$\gamma_{\mathrm{i}}$ : The rate at which AIDS cases are dying (disease related mortality) in Group I.

$\varepsilon$ : The proportion of babies born free of HIV by HIV-infected mothers.

c: The rate at which an individual acquires new (changes) sexual partners.

$\beta$ : The probability of getting an infection from a new sexual partner.

$\beta c$ : The per capita rate of infection of the susceptible by an infective.

$1 / \mathrm{v}_{\mathrm{i}}$ : The mean incubation period of HIV/AIDS in Group i

Let the sexually active adult infects of Group II be divided into:

$\mathrm{I}_{2 \mathrm{~T}}(\mathrm{t})$ - Adult infects who receive treatment

$\mathrm{I}_{2 \mathrm{U}}(\mathrm{t})$ - Adult infects who do not receive treatment (untreated).

Let $\delta$ be a fraction of the infected adults who receive treatment, and $\mathrm{v}_{2}$ and $\mathrm{v}_{2}^{\prime}$, be the rates of progression for $\mathrm{I}_{2 \mathrm{U}}(\mathrm{t})$ and $\mathrm{I}_{2 \mathrm{~T}}(\mathrm{t})$ respectively. Since treatment prolongs incubation period ${ }^{[17,22,23]}$, we have $v_{2}^{\prime}<v_{2}$. Then the two-age group model of treatment of adult infects is described by the following system of equations:

Group I: (0 - $a$ years $)$

$$
\begin{aligned}
& \frac{\mathrm{dS}_{1}}{\mathrm{dt}}=-\mu_{1} \mathrm{~S}_{1}+\lambda\left[\varepsilon\left(\mathrm{I}_{2 \mathrm{~T}}+\mathrm{I}_{2 \mathrm{U}}\right)+\mathrm{S}_{2}\right]- \\
& \mathrm{p} \lambda\left[\varepsilon\left(\mathrm{I}_{2 \mathrm{~T}}(\mathrm{t}-\mathrm{a})+\mathrm{I}_{2 \mathrm{U}}(\mathrm{t}-\mathrm{a})\right)+\mathrm{S}_{2}(\mathrm{t}-\mathrm{a})\right] \\
& \frac{\mathrm{dI}}{\mathrm{dt}}=-\left(\mu_{1}+\mathrm{v}_{1}\right) \mathrm{I}_{1}+\lambda(1-\varepsilon)\left[\mathrm{I}_{2 \mathrm{~T}}+\mathrm{I}_{2 \mathrm{U}}\right] \\
& \frac{\mathrm{dA}}{\mathrm{dt}}=\mathrm{v}_{1} \mathrm{I}_{1}(\mathrm{t})-\gamma_{1} \mathrm{~A}_{1}(\mathrm{t})
\end{aligned}
$$

Group II: (aged $a$ and beyond years)

$$
\begin{aligned}
& \frac{\mathrm{dS}_{2}}{\mathrm{dt}}=-\beta \mathrm{c} \frac{\mathrm{S}_{2}\left(\mathrm{I}_{2 \mathrm{~T}}+\mathrm{I}_{2 \mathrm{U}}\right)}{\mathrm{N}_{2}}-\mu_{2} \mathrm{~S}_{2}+ \\
& \mathrm{p} \lambda\left[\varepsilon\left(\mathrm{I}_{2 \mathrm{~T}}(\mathrm{t}-\mathrm{a})+\mathrm{I}_{2 \mathrm{U}}(\mathrm{t}-\mathrm{a})\right)+\mathrm{S}_{2}(\mathrm{t}-\mathrm{a})\right] \\
& \frac{\mathrm{dI}_{2 \mathrm{~T}}}{\mathrm{dt}}=\delta \beta \mathrm{c} \frac{\mathrm{S}_{2}\left(\mathrm{I}_{2 \mathrm{~T}}+\mathrm{I}_{2 \mathrm{U}}\right)}{\mathrm{N}_{2}}-\left(\mu_{2}+\mathrm{v}_{2}^{\prime}\right) \mathrm{I}_{2 \mathrm{~T}}
\end{aligned}
$$

$\frac{\mathrm{dI}_{2 \mathrm{U}}}{\mathrm{dt}}=(1-\delta) \beta c \frac{\mathrm{S}_{2}\left(\mathrm{I}_{2 \mathrm{~T}}+\mathrm{I}_{2 \mathrm{U}}\right)}{\mathrm{N}_{2}}-\left(\mu_{2}+\mathrm{v}_{2}\right) \mathrm{I}_{2 \mathrm{U}}$

$\frac{\mathrm{dA}_{2}}{\mathrm{dt}}=-\left(\mu_{2}+\gamma\right) \mathrm{A}_{2}+\mathrm{v}_{2} \mathrm{I}_{2 \mathrm{U}}+\mathrm{v}_{2}^{\prime} \mathrm{I}_{2 \mathrm{~T}}$

where $\mathrm{p}=\mathrm{e}^{-\mu_{1} \mathrm{a}}$ is the proportion of uninfected children who survive the developmental stage $(0-a$ years), with

$\mathrm{S}_{1}(0)=\mathrm{f}_{1} \mathrm{~N}_{0}, \quad \mathrm{I}_{1}(0)=0=\mathrm{A}_{1}(0)=0$

$N_{O}$ being the total population at the start of the epidemic and $0<f_{1}<1$ is the fraction thatyear children aged $a$ years at the start of the epidemic. Also we have

$$
\begin{aligned}
& \mathrm{N}_{2}(\mathrm{t})=\mathrm{S}_{2}(\mathrm{t})+\mathrm{I}_{2}(\mathrm{t}) \\
& \mathrm{N}_{2}(\mathrm{t})=\mathrm{f}_{2} \mathrm{~N}_{0} \mathrm{e}^{\mathrm{rt}}, \mathrm{t}<0 \\
& \mathrm{~S}_{2}(0)=\left(1-\varphi_{0}\right) \mathrm{f}_{2} \mathrm{~N}_{0} \\
& \mathrm{I}_{2}(0)=\varphi_{0} \mathrm{f}_{2} \mathrm{~N}_{0} \\
& \mathrm{~A}_{2}(0)=0
\end{aligned}
$$

with $0<f_{2}<1$ as the fraction of the population that were adults before the start of the epidemic; $\phi_{0}$ of which were initially HIV infected. $I_{2}(t) \equiv 0$, for $t<0 ; r=\lambda-\mu$ is the intrinsic rate of growth of the population before the epidemic, where $f_{1}+f_{2}=1$.

\section{POSSIBLE EXISTENCE OF EQUILIBRIA}

We analyze the possibility of equilibria in Group II since it is this group that is sexually active and responsible for the spread. We also assume that the AIDS cases in the population, $A_{2}(t)$ can easily be identified (from the full-blown symptoms) and are not associated with sexually and as such do not pose a 
danger in the spread. But their projection will be investigated for identifying the impact it has on the population.

$\operatorname{Let}\left(S_{2}^{*}, I_{2 T}^{*}, I_{2 U}^{*}\right)$ be the equilibrium point. And at this equilibrium let $S_{2}(t-a)$ and $S_{2}(t) \rightarrow S_{2}^{*}$ and both $I_{2}(t-a)$ and $I_{2}(t) \rightarrow I_{2}^{*}$ as $t \rightarrow \infty$.

Then, equations (4) - (6) give

$$
\begin{aligned}
& -\beta \mathrm{c} \frac{\mathrm{S}_{2}^{*}\left(\mathrm{I}_{2 \mathrm{~T}}^{*}+\mathrm{I}_{2 \mathrm{U}}^{*}\right)}{\mathrm{N}_{2}^{*}}-\mu_{2} \mathrm{~S}_{2}^{*}+ \\
& \mathrm{p} \lambda\left[\varepsilon\left(\mathrm{I}_{2 \mathrm{~T}}^{*}+\mathrm{I}_{2 \mathrm{U}}^{*}\right)+\mathrm{S}_{2}^{*}\right]=0 \\
& \delta \beta c \frac{S_{2}^{*}\left(I_{2 T}^{*}+I_{2 U}^{*}\right)}{N_{2}^{*}}-\left(\mu_{2}+v_{2}^{\prime}\right) I_{2 T}^{*}=0 \\
& (1-\delta) \beta c \frac{S_{2}^{*}\left(I_{2 T}^{*}+I_{2 U}^{*}\right)}{N_{2}^{*}}-\left(\mu_{2}+v_{2}\right) I_{2 U}^{*}=0
\end{aligned}
$$

where $N_{2}^{*}=S_{2}^{*}+I_{2 T}^{*}+I_{2 U}^{*}$.

The disease-free equilibrium point $\left(S_{2}^{*}, 0,0\right)$ exists if $p \lambda-\mu_{2}>0$. (And indeed, for the existence of a disease-free susceptible population, the total recruitment into the sexually active adult group $(p \lambda)$ should necessarily be more than the mortality rate in this group).

For the equilibrium point $\left(S_{2}^{*}, I_{2 T}^{*}, 0\right)$; letting $I_{2 U}^{*}=0$ in equations (10), (11) and (12) gives:

$-\beta c \frac{S_{2}^{*} I_{2 T}^{*}}{S_{2}^{*}+I_{2 T}^{*}}-\mu_{2} S_{2}^{*}+p \lambda\left[\varepsilon I_{2 T}^{*}+S_{2}^{*}\right]=0$

$\delta \beta c \frac{S_{2}^{*} I_{2 T}^{*}}{S_{2}^{*}+I_{2 T}^{*}}-\left(\mu_{2}+v_{2}^{\prime}\right) I_{2 T}^{*}=0$

$$
(1-\delta) \beta c \frac{S_{2}^{*} I_{2 T}^{*}}{S_{2}^{*}+I_{2 T}^{*}}=0 .
$$

From equation (15), for $S_{2}^{*}, I_{2 T}^{*}>0$, we note that $\delta=1$. This implies that the equilibrium point $\left(S_{2}^{*}, I_{2 T}^{*}, 0\right)$ must exit if all the infected in the adult population do receive treatment. Then, forError! Bookmark not defined. in the equation (14), together with (13) gives

$\frac{p \lambda-\mu_{2}}{\mu_{2}+v_{2}^{\prime}-\varepsilon p \lambda}>0$

as a condition for the existence of this equilibrium point. This is as a result of $\hat{R}_{0}>1$, for the epidemic to develop where,

$$
\hat{R}_{0}=\frac{\beta c}{\mu_{2}+v_{2}^{\prime}}
$$

is the number of secondary infections by any one HIV infective that receives treatment. The condition given by (16) is satisfied if $\varepsilon p \lambda-v_{2}^{\prime}<\mu_{2}<p \lambda$

For the equilibrium point $\left(S_{2}^{*}, 0, I_{2 U}^{*}\right)$; if we let $I_{2 T}^{*}=0$ in equations (10), (11) and (12) we get

$-\beta c \frac{S_{2}^{*} I_{2 U}^{*}}{S_{2}^{*}+I_{2 U}^{*}}-\mu_{2} S_{2}^{*}+p \lambda\left[\varepsilon I_{2 U}^{*}+S_{2}^{*}\right]=0$

$\delta \beta c \frac{S_{2}^{*} I_{2 U}^{*}}{S_{2}^{*}+I_{2 U}^{*}}=0$

$(1-\delta) \beta c \frac{S_{2}^{*} I_{2 U}^{*}}{S_{2}^{*}+I_{2 U}^{*}}-\left(\mu_{2}+v_{2}\right) I_{2 U}^{*}=0$.

Equation (19), for $S_{2}^{*}, I_{2 U}^{*}>0$, clearly gives $\delta=0$. This implies that the equilibrium point $\left(S_{2}^{*}, 0, I_{2 U}^{*}\right)$ must exit if none of the infected in the adult population receives treatment. This gives

$\frac{p \lambda-\mu_{2}}{\mu_{2}+v_{2}-\varepsilon p \lambda}>0$

as a condition for the existence of this equilibrium point. This is as a result of $R_{0}>1$, for the epidemic to develop where

$R_{0}=\frac{\beta c}{\mu_{2}+v_{2}}$ 
Is the number of secondary infections by any one HIV infection in the absence of treatment.

Clearly, $\quad \hat{R}_{0}$ given by equation (17) is great equation a $R_{0}$ given by the incubation period $v_{2}^{\prime}<v_{2}$ since treatment prolongs incubation period.

The endemic equilibrium point $\left(s_{2}^{*}, I_{2 T}^{*}, I_{2 U}^{*}\right)$ exists when $0<\delta<1$. Solving equations (10), (11) and (12) simultaneously for $S_{2}^{*}, I_{2 T}^{*}, I_{2 U}^{*}>0$ gives the following situations:

The first case is when $p \lambda-\mu_{2}=0, \varepsilon p \lambda-\left(\mu_{2}+v_{2}\right)=0$ and

$\varepsilon p \lambda-\left(\mu_{2}+v_{2}^{\prime}\right)=0$; which conditions may not all be possible to fulfill by any set of parameters and moreover the condition $p \lambda=\mu_{2}$ is itself not desirable. This would then imply that there is no nontrivial endemic equilibrium, as the only alternative in this case is to haveError! Bookmark not defined. and $I_{2 U}^{*}=0$.

The second case is a possibility of having $p \lambda-\mu_{2}>0, \varepsilon p \lambda-\left(\mu_{2}+v_{2}\right)<0$ and

$\varepsilon p \lambda-\left(\mu_{2}+v_{2}^{\prime}\right)>0$. This gives $\varepsilon p \lambda-v_{2}<\mu_{2}<\varepsilon p \lambda-v_{2}^{\prime}$ as a possible condition for the existence of the positive endemic equilibrium.

The third case is having $p \lambda-\mu_{2}>0, \varepsilon p \lambda-\left(\mu_{2}+v_{2}\right)<0$ and

$\varepsilon p \lambda-\left(\mu_{2}+v_{2}^{\prime}\right)<0 . \quad$ This

gives $\varepsilon p \lambda-v_{2}^{\prime}<\mu_{2}<p \lambda$ as another possible condition for the existence of the endemic equilibrium.

If $p \lambda-\mu_{2}<0$ then $\varepsilon p \lambda-\left(\mu_{2}+v_{2}\right)<0$ and $\varepsilon p \lambda-\left(\mu_{2}+v_{2}^{\prime}\right)<0$. So in this case the endemic equilibrium is not likely to exist. But as mentioned in the first case above, given any set of parameter, it is very unlikely that the conditions in the second and third cases to be simultaneously satisfied. This means that the endemic equilibrium does not exist.

\section{NUMERICAL SIMULATIONS OF THE MODEL}

We present a numerical simulation of model, in the first case varying the proportion treated, then assume effective treatment and we vary the length of the incubation period; and in both cases we shall assume no change in social behavior. We simulate effective treatment taking care of changes in social behavior. The parameters that are used in the numerical simulation of this model are from $^{[23-30]}$. Accordingly we take:

$\mu_{1}=0.03$ average infant mortality rate

$1 / v_{l}=2.5$ years as an average incubation period in children

$\mu_{2}=0.02$ from an average life expectancy of 50 years in Uganda

$1 / v_{2}=8$ years as an average incubation period in adults

$\lambda=0.05$ approximate birth rate in Uganda

$\varepsilon=0.7$ the proportion of children born uninfected by HIV-infected mothers

$\gamma_{2}=0.4 \quad 2.5$ years being the average number of years it takes one who has shown full-blown AIDS symptoms to die of the disease ${ }^{[28,30]}$

$a=12$ years at which an individual becomes sexually mature

$\beta c=0.433$ deduced from the doubling time of 1.5 years during the early stages of infection

The value of $p$ is computed from $p=e^{-\mu_{1} a}$.

Treatment of varying proportions of adult ineffective with no behavioral change: The parameter $\delta$ in equations (4) and (5) is the proportion of the adult infects who receive treatment. In this simulation, we vary this proportion to study the effect of treating a small number, an average number and a big number of HIV-infected on the spread of the epidemic. We look at the effect of treatment of HIV-infected on the susceptible children, susceptible adults, infected children and infected adults. The different proportions of the adult infects who are assumed receiving treatment, used in this simulation are $\delta=0.0,0.3,0.5$, 0.75 and 0.98 .

Fig. 1a to $1 \mathrm{~d}$ are a simulation of the model given by equations (1)-(7) as $\delta$ varies. It is noted that when you treat a small proportion of adult HIV infects (say $\delta=0.0,0.3$ and 0.5 ), we get fewer number of infected children and less adult infects. And when a bigger proportion of adult infects (say, $\delta=0.75$ and 0.9 ,) is treated we get more infected children in the population, many more adult infects in the population (Fig. 1c and 1d). The increase in the number of adult HIV infects with increased treatment is due to the fact that treatment lengthens the lives of the ineffective and as such those who would have died of AIDS do become healthier and continue to produce. This also explains the increase in the number of HIV-infected children in the population, with increased treatment of adult HIV infects. This is in agreement with Becker and Egerton ${ }^{[12]}$ and in Gupta et $a l .{ }^{[15]}$, in which they note that therapy lengthens the incubation period thereby reducing on the AIDS incidence rate and increasing the infectious period. 

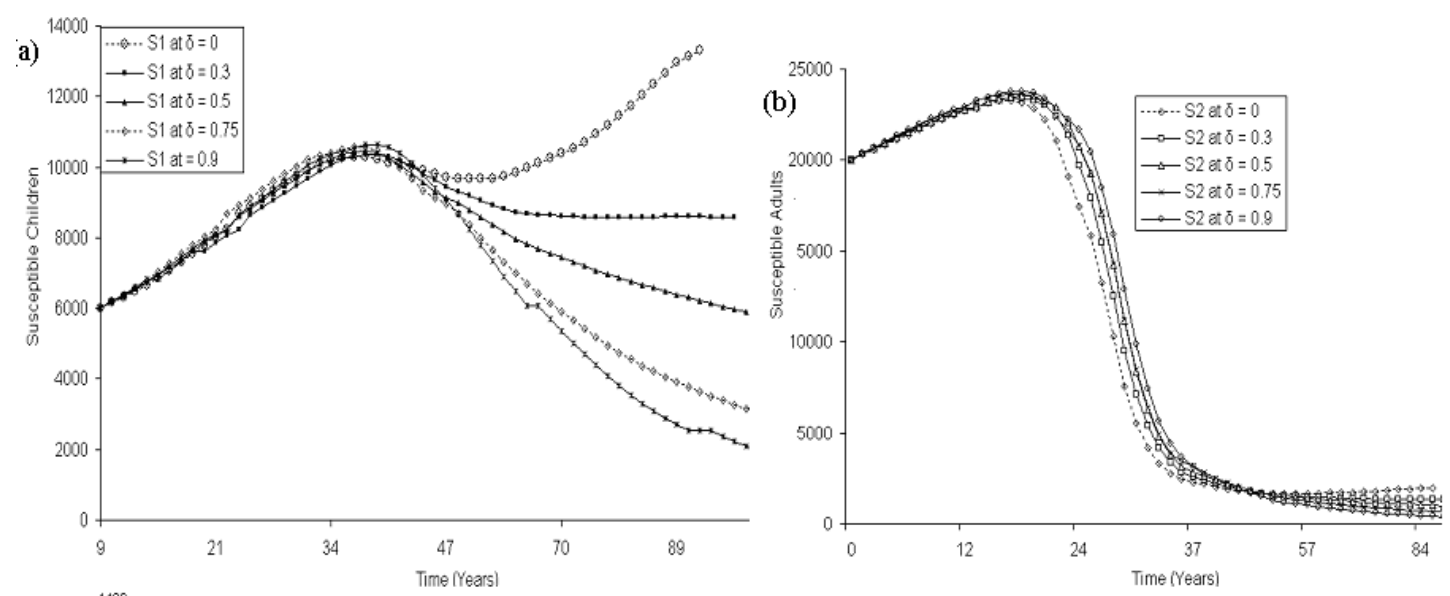

(c)

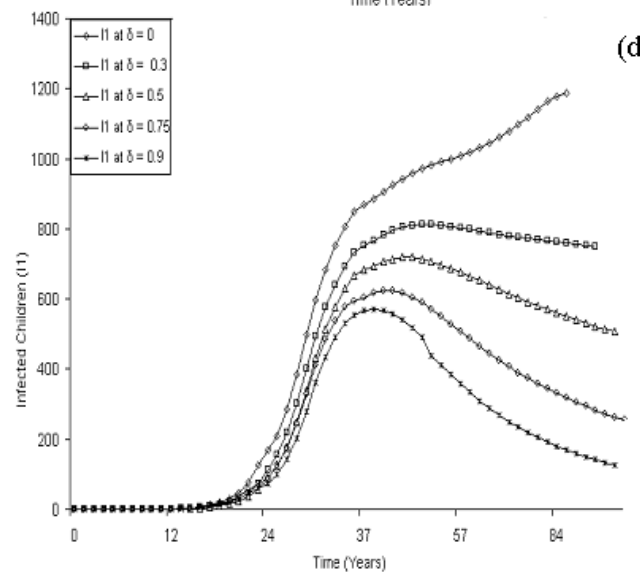

(d)

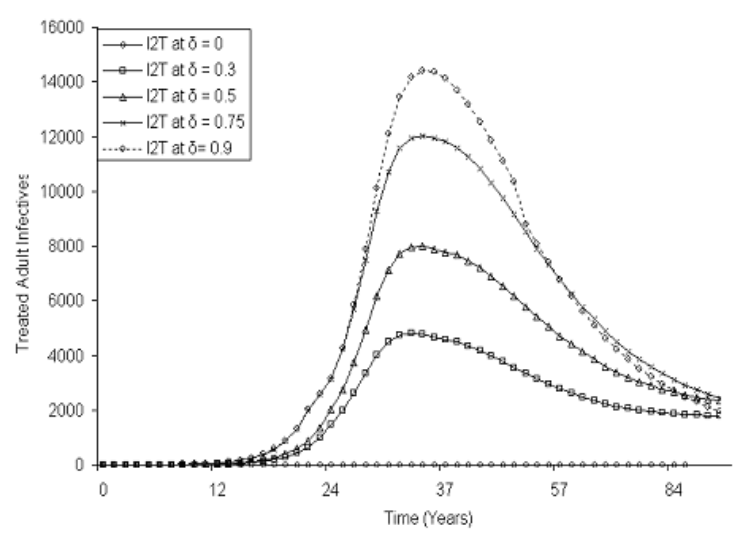

Fig. 1: Treating a proportion $\delta$ of HIV victims: Its effect on susceptible children $S_{1}$, susceptible adults $\mathrm{S}_{2}$, infected children $\mathrm{I}_{l}$ and treated adult infects $\mathrm{I}_{2 T}$

From Fig. 1a and $1 \mathrm{~b}$ treatment of adult HIV infects has little difference it causes in susceptible children and susceptible adults in the population. This is because the treatment of HIV-infected of opportunistic infections is not healing the infected. However, there is a slight increase in the number of children susceptible to continued increased treatment. This is because, as already mentioned, increased treatment leads to increased number of adult infects in the population and a big proportion of children born to HIV-infected mothers are not infected. This eventually leads to such an increase in the number of susceptible children in the population as is noted in Fig. 1a. Although the simulation shows insignificant differences in susceptible populations, it is clear from Fig. 1a and $1 \mathrm{~b}$ that both the children and the adult susceptible populations will be wiped out by the epidemic.

Effective Treatment of adult ineffective with no behavioral change: Since treatment of HIV victims prolongs the incubation period (the length of time that an infected person takes to develop full-blown AIDS symptoms) we look at the effect of lengthening the average incubation period (due to treatment) on the susceptible children, infected children, susceptible adults and infected adults in the population. The average incubation periods of 10, 12 and 14 years are used in equations (2)-(7) of the model with an assumption that a big proportion of these adult infects are receiving treatment. Therefore we have fixed $\delta=$ 0.98 and we are varying the parameter $v_{2}$, to take on values $1 / 10,1 / 12$ and 1/14 corresponding to the values of the average incubation periods. It is also assumed that there is no behavioral change in the population. From Fig. 2a-2d it is noted that the longer the incubation period the bigger the number of infected children Fig. 2c, the bigger the number of susceptible children Fig. 2a, the bigger the number of adult infects in the population (Fig. 2d) and subsequent the more the AIDS cases in the population. As noted earlier, effective treatment of HIV adult ineffective does not have much significance on the number of susceptible children and adults as shown in Fig. 2a and 2b. This is because the treatment of HIV-infected is not healing them from the disease. An increase in the number of infected children and in the number of adult ineffective and hence the number of AIDS cases in the population, associated with prolonged incubation periods, as shown in Fig. 2c and $2 d$ is an indicator that 
effective treatment of adult ineffective results into more spread of the epidemic.

Effective treatment of adult infectives: Varying levels of behavioral change: Here, we study the trend of the spread of the epidemic, in relation to effective treatment that is accompanied by objective measures geared towards changing one's social behavior.

In the model, $c$ is the average rate at which individuals change (or acquire new) sexual partners. Control measures such as abstinence from sex and Zero grazing or sticking to one sexual partner can reduce on $c$ and consequently reduce the probability $\beta$ of getting infected. The combination of these leads to smaller values of $\beta c$. Whereas the use of condoms may reduce on the probability of getting infected $\beta$, it may in turn lead to increased acquisition of new sexual partners well knowing that one is protected. This measure may however lead to large values of $\beta c$. Small values of $\beta c$ imply a positive change in one's social behaviors and a big value of $\beta c$ indicates little or no change in one's social behavior.

Since treatment lengthens the incubation period we fix an average incubation period to 14 years (assuming this could be the longest average period an HIVinfected adult, once treated, would take to finally show full-blown AIDS symptoms) and assume effective

(a)

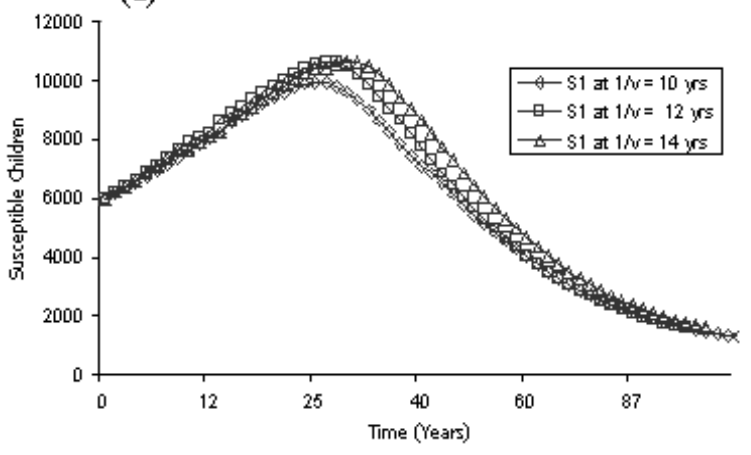

(c)

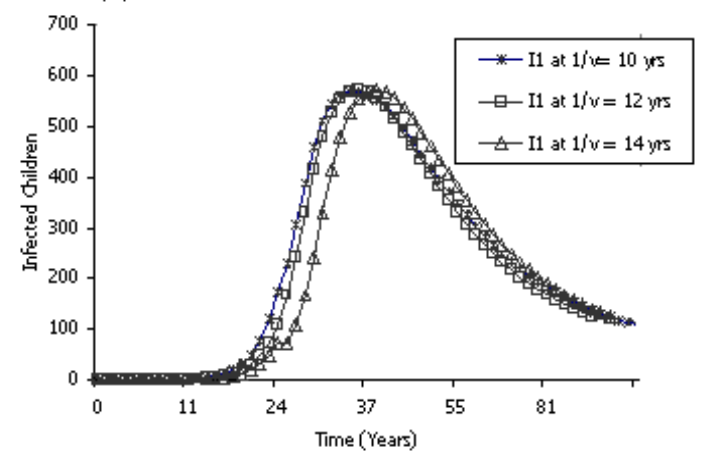

treatment (by fixing $\delta=0.98$ ). Taking the values of $\beta c=$ $0.01,0.2,0.4,0.5$ (with smaller values taken to mean a positive behavioral change and bigger values to mean little or no change in one's social behaviors) we have a simulation of the model as shown in Fig. 3a-3d.

With effective treatment of HIV-infected, where a large proportion of the adult infected population seeks medical treatment of most of the opportunistic infections, we note from Fig. $3 a$ and $3 b$ that effective treatment coupled with a positive change in one's social behaviors (i.e. Smaller values of $\beta c$ say 0.01 ), increases on the number of susceptible children and sexually active susceptible adults in the population. But with less change in one's social behaviors (i.e. Bigger values of $\beta c$ say 0.2 and 0.4 ) there is a notable early decrease in the number of children and adult susceptibles in the population. However, with $\beta c$ as small as possible, implying that there is a positive change in one's social behavior, (or such a treated person has not gone back to re-infect others as a result of becoming healthier) we note in Fig. $3 \mathrm{a}$ and $3 \mathrm{~b}$ that both children and adult susceptible population grows without a decline. This shows that treatment accompanied by measures such as counseling the treated HIV patients to refrain from unprotected sexual activities will eventually reduce and/or eradicate the epidemic in the long run.

(b)

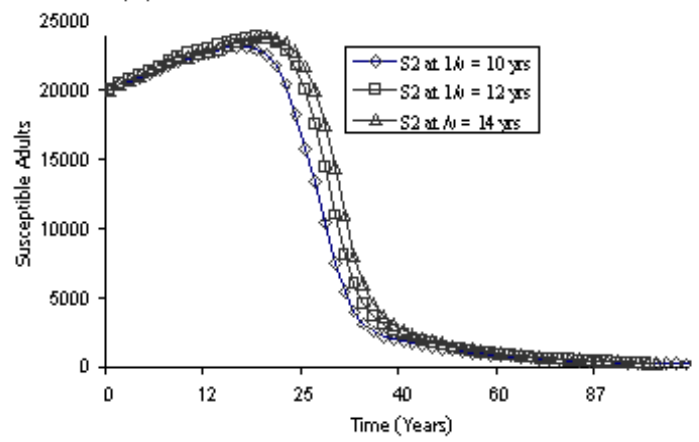

(d)

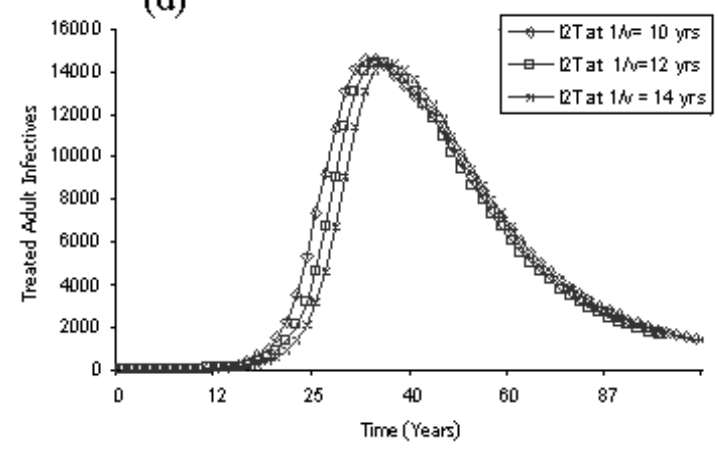

Fig. 2: Treating a proportion $\delta$ of HIV victims: Its effect on susceptible children $S_{1}$, susceptible adults $S_{2}$, infected children $I_{l}$ and treated adult infects $I_{2 T}$ 

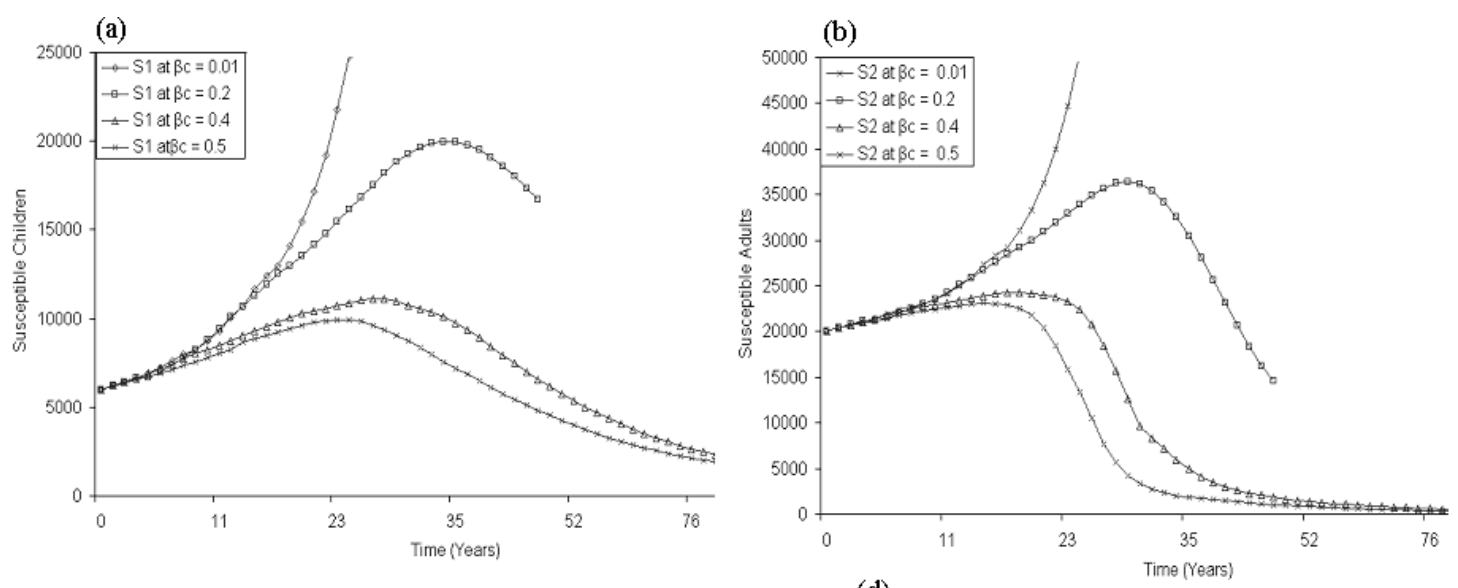

(c)
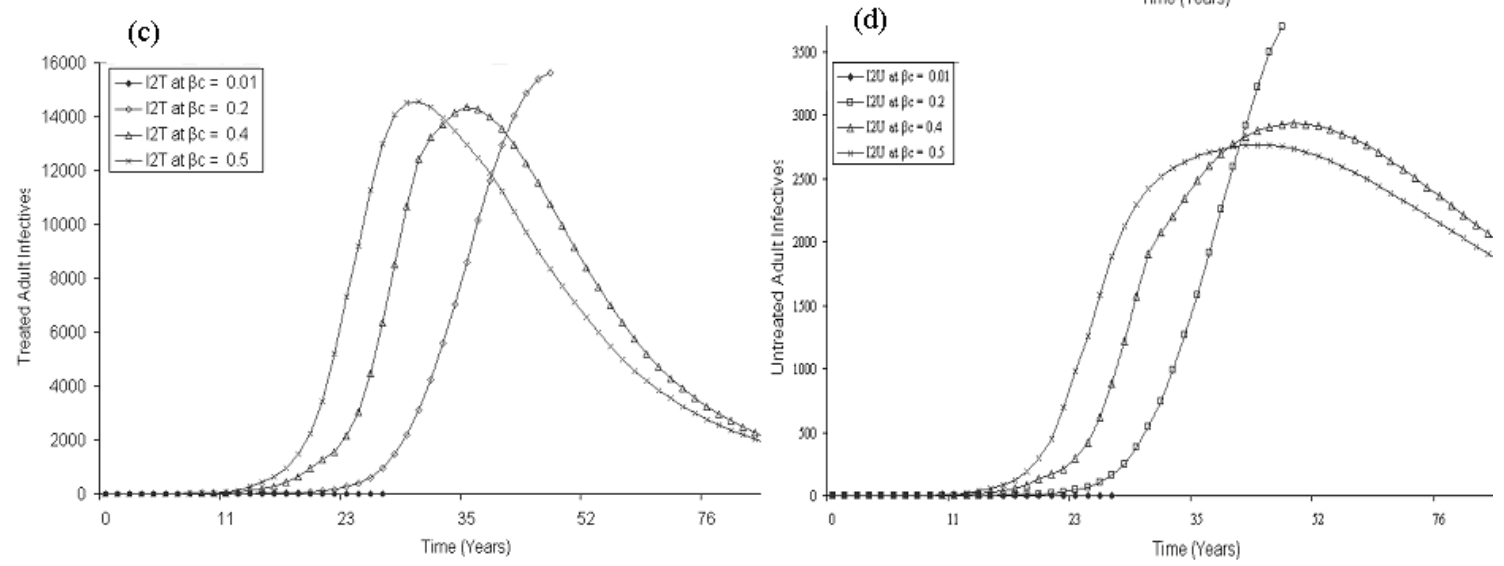

Fig. 3: Treating a proportion $\delta$ of HIV victims: Its effect on susceptible children $S_{1}$, susceptible adults $S_{2}$ and treated adult infects $I_{2 T}$ and untreated adult infects $I_{2 U}$

Figure $3 \mathrm{c}$ and $3 \mathrm{~d}$, show that with little or no change in one's social behaviors, the number of infective (both treated and untreated) in the population will grow earlier than when there is a positive change. As a result of this, we would expect early progression to AIDS.

\section{RESULTS AND DISCUSSION}

In the analysis of the model, it is clear that both the disease-free and endemic equilibria in a population with HIV/AIDS may be hard to attain with treatment. From the expressions for the number of secondary infections, $R_{0}=2.9862$ in the absence of treatment and $\hat{\mathrm{R}}_{0}=5.2485$ when we treat adult infects in the population. This is evidence that treatment increases the spread of the epidemic as the number of secondary infections increases more than when there is no treatment. A decrease in the value of the parameter $\beta c$ that governs the force of infection shows a reduced numerical value for the number of secondary infections with treatment and with no treatment. For example when we take $\beta c=0.1$, a rather small value that indicates a positive change in social behavior, we have the corresponding values of $R_{0}=0.6896$ for no treatment of infective and $\hat{\mathrm{R}}_{0}=1.2121$ for treatment of adult infects. Table 1 gives the summary of the number of secondary infections from different treatment scenarios vis-à-vis levels of behavioral change. It is noted that the smaller the values of $\beta c$ (high rate of behavioral change), the fewer the number of secondary infections in the population and the bigger the values of $\beta c$ of which implies little of behavior in social behavior) the more the number of secondary infections. Also we note that the number of secondary infections increases with treatment of adult infects and big values of $\beta c$. This is evidence that treatment that is not accompanied by objective measures would lead to more re-infections in the population and hence increase the rate of the spread of the epidemic. And when treatment is accompanied by a change in social behavior then the spread will be computed as the number of secondary infections will be reduced.

The treatment increases the length of the incubation period of HIV without significantly reducing infectiousness and this will necessarily increase the prevalence of the infection, unless accompanied by appropriate levels of behavior change ${ }^{[15]}$. On the question of who benefits from treatment of HIV infects: an individual or a community? 
Table 1: The number of secondary infections in treatment of HIVinfected vis-à-vis the change in social behavior No. of secondary Infections

\begin{tabular}{lll} 
& $R_{0}$ & $\hat{\mathrm{R}}_{0}$ \\
\hline$B c=0.1$ & 0.6896 & 1.2121 \\
$B c=0.2$ & 1.3793 & 2.4242 \\
$B c=0.433$ & 2.9862 & 5.2485 \\
$B c=0.6$ & 4.1379 & 7.2727 \\
$B c=0.8$ & 5.5172 & 9.6970 \\
\hline
\end{tabular}

It is clear from the study that a sick individual would benefit first since treatment lengthens one's life to live, but this would be beneficial to the community if treatment is accompanied by a positive change in social behavior, by the individual that has received treatment. If treatment coverage and efficacy are high and with a high positive change in social behavior there is a big chance that the epidemic would even come to an end.

\section{ACKNOWLEDGEMENT}

The author would like to thank the anonymous referee for the useful comments and NUFU for financial support.

\section{REFERENCES}

1. Cello, J.P., 1988. Gastrointestinal manifestation of AIDS. In: M. A. Sande, P.A. Volberding (Eds). The Medical Management of AIDS, Philadelphia, Saunders, pp: 141.

2. Curtis, L., 1986. Noninvasive tests in the diagnostic evaluation for $P$. carinii pneumonia in patients with or suspected of having AIDS. Am. Rev. Respir. Dis., 133A: 182.

3. Davis, S.D., 1987. Intrathoracic Kaposi sarcoma in AIDS patients: Radiographic pathologic correlation. Radiology, 163: 495.

4. De CocK, K.M., 1995. Screening for tuberculosis and HIV in resource-poor countries. Lancet, 348: 873-4.

5. Handwerger, S., 1987. Tuberculosis and the acquired immunodeficiency syndrome in a New York City hospital: 1978-1985. Chest, 91:176.

6. Klein, R.S., 1984. Oral candidaisis in high-risk patients as the initial manifestation of the acquired immunodeficiency syndrome. N. Engl. J. Med., 311: 354.

7. Allegra, C.J., 1987. Trimetrexate, a novel and effective agent for treatment of Pneumocystis carinii pneumonia in patients with the acquired immunodeficiency syndrome. N. Engl. J. Med., 317: 978.

8. Drew, W.L., 1988. Herpesvirus infections (caused by cytomegalovirus, herpes simplex virus, varicella-zoster virus): How to use ganciclovir (DHPG) and acyclovir. In: M.A.Sande, P.A. Volberding (Eds.) The Medical Management of AIDS, Philadelphia, Saunders, pp: 271.
9. Hirsch, M.S., 1988. AIDS CommentaryAzidothymidine. J. Infect. Dis., 157:427.

10. Richman, D.D., 1987. The toxicity of azidothymidine (AZT) in the treatment of patients with AIDS and AIDS-related complex-A doubleblind, placebo-controlled trial. N. Engl. J. Med., 317:192.

11. Anderson, R.M., 1988. The epidemiology of HIV infection: Variable incubation period plus infectious periods and heterogeneity in sexual activity. J. Roy. Stat. Soc. A, 151: 66-93.

12. Becker, N.G. and L.R. Egerton, 1994. A transmission model for HIV with application to the Australian epidemic. Math. Biosci., 119: 205-224.

13. De Boer, R.J. and C.A.B. Boucher, 1996. AntiCD4 therapy for AIDS suggested by mathematical models. Pro. Roy. Soc. Lond. B, 263: 899-905.

14. De Valle, S., A.M. Evangelista, M.C. Velasco, C.M. Kribs-Zaleta and S.-F. Hsu Schmitz, 2004. Effects of education, vaccination and Treatment on HIV transmission in homosexuals with heterogeneity. Math. Biosc., 187: 111-133.

15. Gupta, S., R.M. Anderson and R.M. May, 1993. Mathematical Models and the design of public health policy: HIV and antiviral therapy. SIAM Rev., 35: 1-16.

16. Johnson, A.M., 1988. Social and behavioral aspect of HIV epidemic- A review. J. Roy. Stat. Soc. A, 151: 99-114.

17. McLean, A.R., V.C. Emery, A. Webster and P.D. Griths, 1991. Population dynamics of HIV within an individual after treatment with Zidovudine. AIDS, 5: 485-489.

18. McLean, A.R. and S.D.W. Frost, 1995. Zidovudine and HIV: Mathematical models within-host population dynamics. Rev. Med. Virol., 5: 141-147.

19. Mugisha, J.Y.T. and L.S. Luboobi, 2002. The Effect of Treatment of HIV/AIDS patient in a Two-Age Groups Population. SAMSA J., 2: 106130.

20. Porto, T.C., J.O. Lloyd-Smith, K.L. Gross and A.P. Galvan, 2005. The effect of treatment on pathogen virulence. J. Theor. Biol., 233: 91-102.

21. Velasco-Hernandez, J.X., F. Brauer and C. Castillo-Chavez, 1996. Effects of treatment and prevalence-dependent recruitment on the dynamics of a fatal disease. IMA J. Math. Appl. Med. Biol., 13: 175-192.

22. Kramer, I. 1991. Is AIDS an invariably Fatal Disease? A model Analysis of AIDS Survival Curves. Mathl. Comput. Modelling, 15: 1-19.

23. Luboobi, L.S., 1994. A Three-Age Groups Model for the HIV/AIDS epidemic and the Effect of Medical/Social Intervention. Mathl. Comp. Modelling, 19: 91-105. 
24. Aids Control in Uganda. A Multi-sectoral Approach. AIDS CONTROL PROGRAMME , February 1993 Report.

25. Anderson, R.M. and A.R. McLean, 1988. Possible demographic consequences of AIDS in Developing countries. Nature, 332: 228-234.

26. Luboobi, L.S., 1991. Mathematical Models for the dynamics of the AIDS epidemic. In: Patel M.S. and Nokoe S. (Eds). Biometric for development. ICIPE, Science Press, Nairobi, pp: 76-83.

27. May, R.M., R.M. Anderson and A.R. McLean, 1988. Possible demographic consequences of HIV/AIDS epidemic. 1. Assuming HIV infection always leads to AIDS. Math. Biosc., 90: 475-505.
28. McLean, A.R. and S.M. Blower, 1993. Imperfect vaccines and herd immunity to HIV. Proc. Roy. Soc. Lond. (B), 253: 9-13.

29. Mugisha, J.Y.T., 1992. Mathematical Models for the Dynamics of HIV/AIDS epidemic for a Three Age-Groups Population. An M.Sc. Dissertation, Department of Mathematics, Makerere University.

30. Porco, T.C. and S.M. Blower, 1998. Designing HIV Vaccination Policies: subtypes and crossimmunity. Interfaces, 28: 167-190. 